Draft VERSiOn July 30, 2018

Preprint typeset using $\mathrm{L}_{\mathrm{T}} \mathrm{EX}$ style emulateapj v. 5/2/11

\title{
THE IMPRINT OF MASSIVE BLACK-HOLE MERGERS ON THE CORRELATION BETWEEN NUCLEAR STAR CLUSTERS AND THEIR HOST GALAXIES
}

\author{
Fabio Antonini ${ }^{1}$, Enrico Barausse ${ }^{2,3}$ \& Joseph $\operatorname{Silk}^{2,3,4,5}$ \\ (1) Center for Interdisciplinary Exploration and Research in Astrophysics (CIERA) and Department of Physics and Astrophysics, \\ Northwestern University, Evanston, IL 60208
}

(2) Sorbonne Universités, UPMC Univ Paris 06, UMR 7095, Institut d'Astrophysique de Paris, F-75014, Paris, France (3) CNRS, UMR 7095, Institut d'Astrophysique de Paris, F-75014, Paris, France

(4) Laboratoire AIM-Paris-Saclay, CEA/DSM/IRFU, CNRS, Universite Paris Diderot, F-91191 Gif-sur-Yvette, France (5) Department of Physics and Astronomy, Johns Hopkins University, Baltimore MD 21218, USA

Draft version July 30, 2018

\begin{abstract}
A literature compilation of nuclear star cluster (NSC) masses is used to study the correlation between global and nuclear properties. A comparison of observational data to the predictions of semianalytical galaxy formation models places constraints on the co-evolution of NSCs, massive black holes (MBHs) and host galaxies. Both data and theoretical predictions show an increased scatter in the NSC scaling correlations at high galaxy masses, and we show that this is due to the progressively more efficient ejection of stars from NSCs caused by MBH binaries in more massive stellar spheroids. Our results provide a natural explanation of why in nucleated galaxies hosting a $\mathrm{MBH}$, the ratio $\left(M_{\mathrm{NSC}}+M_{\mathrm{MBH}}\right) / M_{\text {bulge }}$ (with $M_{\text {bulge }}$ the host spheroid's mass) shows significantly less scatter than $M_{\mathrm{NSC}} / M_{\text {bulge }}$, and suggest that the formation of MBHs and NSCs are not mutually exclusive, as also supported by observations of co-existing systems. Both MBHs and NSCs represent generic products of galaxy formation, with NSCs being destroyed or modified by the merger evolution of their companion MBHs.

Subject headings: galaxies: Milky Way Galaxy - Nuclear Clusters - stellar dynamics
\end{abstract}

\section{INTRODUCTION}

Observations over the last two decades have unveiled the existence of compact stellar nuclei at the centers of most intermediate and low luminosity galaxies (Carollo et al. 1998; Böker et al. 2002. den Brok et al. 2014; Georgiev \& Böker 2014). With masses of order $0.1 \%$ the stellar mass of the host galaxy and typical radii of only a few parsecs, these clusters, often referred to as NSCs, are the densest stellar systems observed in the Universe (e.g., Walcher et al. 2005; Côté et al. 2006; Carson et al. 2015).

The masses of NSCs $\left(M_{\mathrm{NSC}}\right)$ obey fairly tight correlations with properties of the host galaxies such as the bulge velocity dispersion $(\sigma)$ and mass ( $M_{\text {bulge }}$ ) (Ferrarese et al. 2006a). A number of authors have shown that these correlations are much shallower than the corresponding ones for MBHs. Graham (2012) finds that NSC masses obey $M_{\mathrm{NSC}} \sim \sigma^{2}$, while the masses of MBHs, $M_{\mathrm{MBH}}$, follow a much steeper scaling relation $M_{\mathrm{MBH}} \sim \sigma^{5}$ (Ferrarese \& Ford 2005). It is intriguing that for galaxies containing both a NSC and a $\mathrm{MBH}$, the ratio $M_{\mathrm{CMO}} / M_{\text {bulge }}$ (with $M_{\mathrm{CMO}}=$ $M_{\mathrm{MBH}}+M_{\mathrm{NSC}}$ the total mass in central massive objects) shows less scatter than either $M_{\mathrm{MBH}} / M_{\text {bulge }}$ or $M_{\mathrm{NSC}} / M_{\text {bulge }}$ Kormendy \& Ho 2013). The facts that both NSCs and MBHs are found to follow tight correlations with their host galaxy properties, that they are found to coexist in some galaxies, and that in these galaxies they have comparable masses, point toward a picture where NSCs and MBHs are both generic by-products of galaxy formation, and the growth mechanisms of NSCs and MBHs are related to one another and to the host galaxy evolution.
We have developed a semi-analytical model following the formation and evolution of galaxies, $\mathrm{MBHs}$ and NSCs along cosmic history. Previous calculations assumed NSC formation to take place in isolated galaxies (e.g., Agarwal \& Milosavliević 2011; Antonini 2013; Gnedin et al. 2014; Arca-Sedda \& Capuzzo-Dolcetta 2014; Aharon \& Perets 2015), thus neglecting the possible role of galaxy mergers, as well as in-situ star formation processes. Also, these early idealized attempts did not explore the possible interplay between $\mathrm{MBH}$ and NSC evolution. Our model sheds light on exactly these points, i.e. it allows us to assess for the first time the role of galaxy mergers, MBH mergers and nuclear star formation on the formation and evolution of NSCs.

\section{METHOD AND MODEL}

The backbone of this study is the semi-analytical galaxy formation model of Barausse (2012) (with the improvements described in Sesana et al. (2014)). This model tracks the evolution of baryonic structures (both in central galaxies and in satellites) along dark matter merger trees produced with an extended Press-Schechter formalism, modified to reproduce the results of $N$-body simulations of dark matter halos (Parkinson et al. 2008). The baryonic structures ultimately originate from the hot, largely unprocessed intergalactic medium, which cools to form cold gaseous galactic disks. These undergo quiescent star formation, which chemically enriches the interstellar medium and gives rise to stellar disks. Both stellar and gaseous disks are disrupted by bar instabilities - giving rise to pseudo-bulges that undergo a quiescent disk-like star-formation process - and by major mergers - which form classical bulges with violent star-formation bursts, possibly triggered by turbu- 

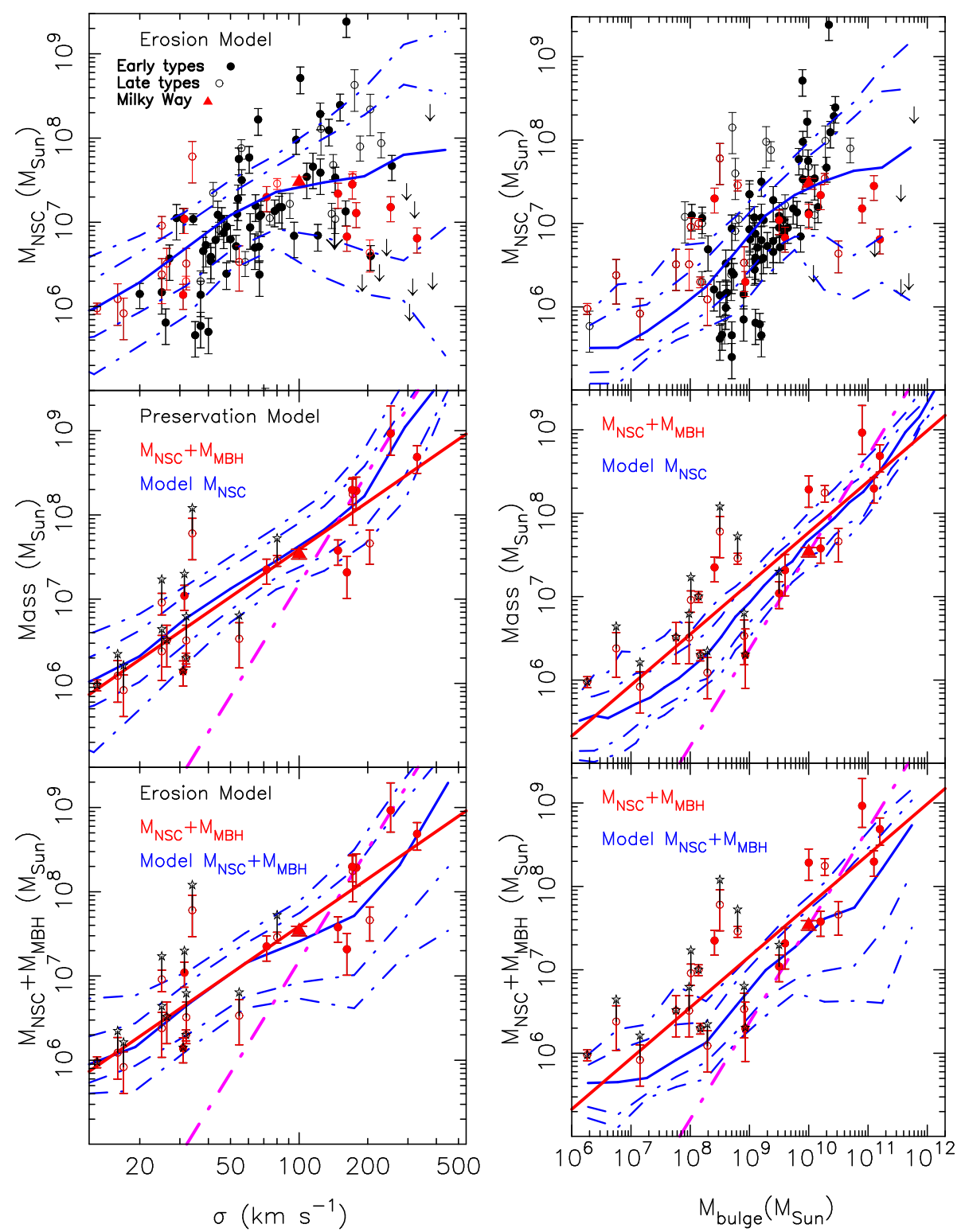

FIG. 1.- Comparison of our model to the observed NSC scaling relations. The upper and lower panels present respectively the predicted $M_{\mathrm{NSC}}$ and $M_{\mathrm{NSC}}+M_{\mathrm{MBH}}$ for the "erosion" model, which accounts for the mass deficit caused by MBH binaries, while the middle panels give $M_{\text {NSC }}$ for the "preservation" model, where this effect is absent. Blue lines are the median correlations predicted by our model; dot-dashed lines represent regions containing $70 \%$ and $90 \%$ of the NSCs produced in our models at a given stellar mass. Arrows are upper limits to the mass of NSCs. Red symbols denote galaxies with observationally constrained MBH masses; the red symbols in the different panels correspond to the same subset of galaxies, but in the upper panels they give $M_{\mathrm{NSC}}$, in the bottom and middle panels they give $M_{\mathrm{NSC}}+M_{\mathrm{MBH}}$. For galaxies with only an upper limit to $\mathrm{MBH}$ mass, we set $M_{\mathrm{MBH}}=0$, but star symbols show how these points would move if we were to assume MBH masses equal to these upper limits. The red lines are fits to the total NSC+MBH masses, while dot-dashed-purple lines show for comparison the MBH - host galaxy scaling correlations (Kormendy \& Ho| 2013).

lent gas flows. Besides further enriching the interstellar medium, bar instabilities and (mainly) star-bursts are assumed to funnel cold, chemically enriched gas into a low-angular momentum nuclear reservoir, available for accretion onto the central MBH (Granato et al. 2004; Haiman et al. 2004). Possible mechanisms capable of removing angular momentum from the gas are shocks (caused e.g. by mergers) or radiation drag from starbursts (Umemura 2001; Granato et al. 2004), and we thus assume a linear correlation between the feeding of the nuclear reservoir and the star-formation rate in the bulge (or pseudo-bulge). The MBHs may form from either high-redshift seeds arising from Pop III stars (i.e. $\sim 100 M_{\odot}$ "light" seeds; Madau \& Rees 2001) or from the remnants of protogalactic disks (i.e. $\sim 10^{5} M_{\odot}$ "heavy" seeds; Koushiappas et al. 2004; Volonteri et al. 2008). In the former case, we allow moderately superEddington accretion onto the massive MBHs so as to better reproduce the quasar luminosity function at high redshift (Madau et al. 2014).

Here, we improve on this model by supplementing it with semi-analytical prescriptions for the formation and evolution of NSCs. These can grow either by local star formation in the nuclear regions (e.g., Seth et al.|2006), or by infall of stellar clusters (Tremaine et al. 1975) toward the center of the galaxy, as a result of dynamical friction.

In more detail, we assume that the nuclear reservoir 
from which the $\mathrm{MBH}$ accretes also undergoes quiescent disk-like star formation - for which we adopt the same star-formation prescription as in galactic disks and pseudobulges, i.e. that of Krumholz et al. (2009) - and we then assume that the stars formed in this nuclear reservoir contribute to the NSC. Note that even though we model star formation in this nuclear gas reservoir as quiescent, our models also assume that the reservoir's feeding is correlated with star formation in the bulge, and thus mainly with turbulence-driven starbursts following major mergers.

As for the infall of stellar clusters, we assume that the latter form with an efficiency $f_{\mathrm{gc}}$ during star-formation episodes in both disks and bulges/pseudo-bulges, and with initial mass function $\mathrm{d} n / \mathrm{d} M \propto M^{-2}$ (Bik et al. 2003), between $M=10^{2} M_{\odot}$ and $M=10^{7} M_{\odot}$. Initially, these stellar clusters follow the same spatial distribution as the stars, but after their formation we follow their (average) infall towards the NSC under dynamical friction from both the stars and gas. In doing so, we also account for both tidal heating and evaporation of the stellar clusters due to the galaxy's tidal field, and for the tidal disruption by the $\mathrm{MBH}$ when they approach the center. When the MBH mass becomes $\geq 10^{8-9} M_{\odot}$, the stellar clusters are completely disrupted before reaching the center, and the contribution of stellar cluster infall to NSC growth becomes negligible (Antonini 2013).

Whenever two galaxies merge, their MBHs are assumed to form a binary. Due to the interactions with the stars in the nuclear region, a $\mathrm{MBH}$ binary ejects from the system a mass (in stars) comparable to its own mass (Merritt 2006). Also, when a MBH binary finally merges, the anisotropic emission of gravitational waves imparts a kick (of up to several thousands of $\mathrm{km} / \mathrm{s}$, cf. Campanelli et al. (2007)) to the MBH remnant, and this also removes stars from the nuclear region. We thus model the total mass deficit $M_{\mathrm{ej}}$ caused by the inspiral and merger of $\mathrm{MBH}$ binaries onto the NSC mass by

$$
\begin{aligned}
M_{\mathrm{ej}} \approx & 0.7 q^{0.2} M_{\mathrm{bin}}+0.5 M_{\mathrm{bin}} \ln \left(\frac{a_{\mathrm{h}}}{a_{\mathrm{gr}}}\right) \\
& +5 M_{\mathrm{bin}}\left(V_{\mathrm{kick}} / V_{\mathrm{esc}}\right)^{1.75}
\end{aligned}
$$

where $q \leq 1$ is the binary's mass ratio, $M_{\text {bin }}$ its mass, $a_{\mathrm{h}}$ the semi-major axis when the binary first becomes "hard" (i.e. tightly bound), $a_{\text {gr }}$ the separation at which gravitational-wave emission starts driving the binary's evolution (cf. explicit expressions for $a_{\mathrm{h}}$ and $a_{\mathrm{gr}}$ in Merritt (2013)), $V_{\text {kick }}$ the post-merger kick velocity (computed using the fit to numerical-relativity simulations presented in van Meter et al. (2010) ), and $V_{\text {esc }}$ the escape velocity from the central parts of the galaxy (computed using the bulge and NSC density profiles).

The first term in equation (11) accounts for the mass deficit before the MBH binary becomes hard (Merritt 2006); the second is the mass ejected from $a_{\mathrm{h}}$ to $a_{\mathrm{gr}}$; and the third represents the mass deficit generated as the kicked $\mathrm{MBH}$ heats up the surrounding core (Gualandris \& Merritt 2008).

We also account for the tidal disruption of NSCs by MBHs during galaxy mergers. More precisely, in a merger between a galaxy containing a $\mathrm{MBH}$ and one containing only a NSC, the NSC is tidally disrupted before

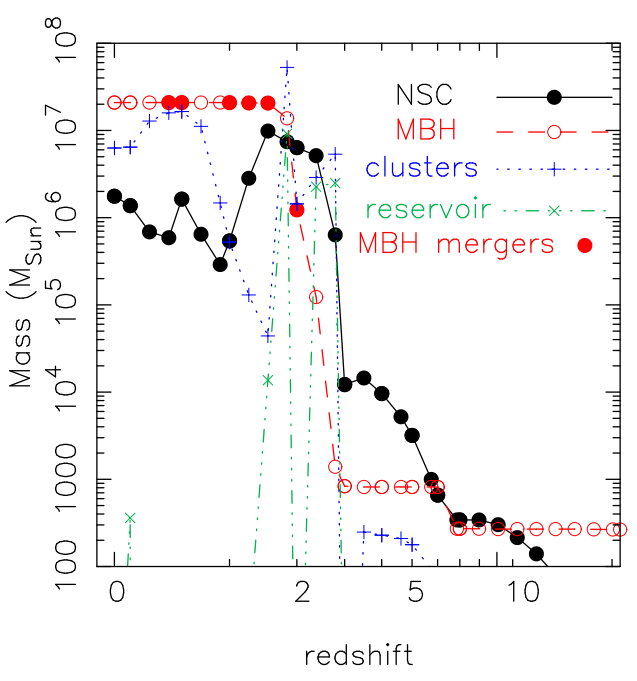

FIG. 2.- Main progenitor evolutionary track of a galaxy whose NSC is partially disrupted by MBH mergers. We show the mass of the NSC, of the MBH, of the central gas reservoir, and the total mass in stellar clusters, as a function of $z$.

reaching the center of the newly formed galaxy, if the MBH mass is $\gtrsim 10^{8-9} M_{\odot}$. This is the same process described above for the infall of stellar clusters.

Next, we present our model's predictions, with the free parameter $f_{\mathrm{gc}}$ set to its Milky Way value, i.e. $f_{\mathrm{gc}} \approx$ 0.07 Kruijssen 2012). We have checked the robustness of our results against the $\mathrm{MBH}$ seed model (i.e. light vs heavy seeds, with several halo occupation numbers), a different value for $f_{\mathrm{gc}} \lesssim 0.2$, a variable $f_{\mathrm{gc}}$ (set to $0.07,0.04$ and 0.5 in disk, quiescent and starburst galaxies respectively; Kruijssen 2012) and other details of our model (i.e. merger-tree resolution, initial redshift of the simulations, AGN feedback prescriptions, etc.). Indeed, we will show that the crucial ingredient to reproduce the observed global to NSC correlations is the scouring effect due to $\mathrm{MBH}$ binaries described by equation (11). More details of our model will be presented elsewhere (Antonini et al. in prep.).

\section{RESULTS}

We describe the results from two realizations of our galaxy-formation model, namely our fiducial model, which accounts for the "NSC erosion" due to $\mathrm{MBH}$ binaries through equation (11); and a "NSC preservation" model where that effect is absent (i.e. we set $M_{\mathrm{ej}}=0$ throughout the entire cosmic history).

Our model's results are compared to a literature compilation of NSC masses. We constructed our sample of NSC objects by combining data from Table 1 of Scott \& Graham (2013), Table 2 of Erwin \& Gadotti (2012), Table 2 of Neumaver \& Walcher (2012) and by estimating NSC masses for the early type galaxies in the Fornax cluster catalog of Turner et al. (2012). The masses of the NSCs in Turner et al. (2012) sample were calculated from the $(g-z)$ colors given in that paper and from the relations of Bell et al. (2003). The galaxy velocity dispersion and bulge mass were also obtained from these papers or, when not available, from the Hyperleda database (Paturel et al. 2003).

The upper panels of Figure 1 compare the observed $M_{\mathrm{NSC}}-\sigma$ and $M_{\mathrm{NSC}}-M_{\text {bulge }}$ relations to our "NSC ero- 

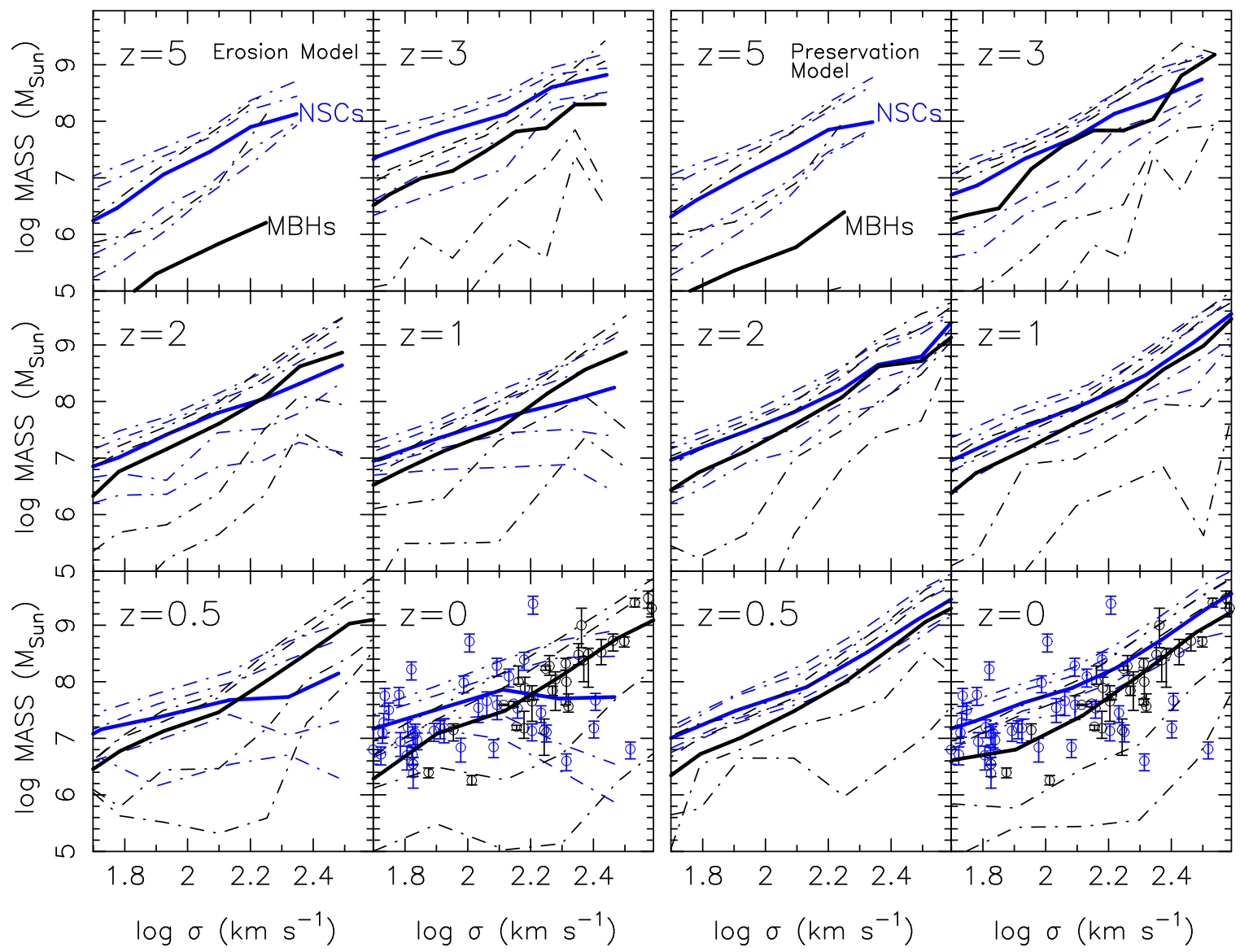

FIG. 3.- Redshift evolution of NSC and MBH $M-\sigma$ relations in our models with (left panels) and without (right panels) mass ejection due to $\mathrm{MBH}$ binaries. The bottom right panels compare our predictions to data. Blue open circles are NSCs; black open circles are MBH masses from Tremaine et al. (2002). Note the different evolution of the NSC mass vs $\sigma$ relation in the two models, at $z<2$. Since reliable MBH mass measurements have been obtained almost exclusively for main early type galaxies (e.g., Ferrarese \& Ford 2005), in this analysis we have neglected MBHs and NSCs in satellite galaxies, and only included galaxies with bulge to total mass ratio $B / T>0.7$, in order to compare with the observed MBH $M-\sigma$ relation. However, if all galaxies were included, the NSC scaling correlations would be very similar to those shown here (see Fig. 1 which was produced by considering all galaxies).

sion" model. We note the excellent agreement of our predictions with observations. In particular, the predicted scaling relations appear to broaden at higher velocity dispersion, as do the data. The broadening of the data appears to be caused by the subsample of galaxies with determined MBH mass (red points in Figure 11). The nuclear to global correlations in these galaxies appear to flatten or even decline for spheroids with higher velocity dispersion, suggesting that the $\mathrm{MBH}$ s have played an important role in affecting the properties of their host NSCs in these galaxies.

The blue lines in the middle panels of Figure 1 shows the scaling relations obtained from the "NSC preservation" model. Note that the broadening at high $\sigma$ 's present in the upper panels disappears. This demonstrates that the broadening of the scaling relations seen in the "NSC erosion" model is a consequence of dynamical ejection of stars from coalescing $\mathrm{MBH}$ binaries. Together with the model's predictions, we plot $M_{\mathrm{CMO}}=$ $M_{\mathrm{NSC}}+M_{\mathrm{MBH}}$ vs $\sigma$ and $M_{\text {bulge, }}$, for nucleated galaxies with measured $\mathrm{MBH}$ mass or an upper limit to it. $\mathrm{MBH}$ masses and upper limits were taken from Table 1 of Erwin \& Gadotti (2012), table 2 of Neumaver \& Walcher (2012), and Table 1 of Graham \& Spitler (2009). The red lines show fits to these data. As can be seen, when the disruptive effect of $\mathrm{MBH}$ binaries is not included, our model recovers the fitted functional form of the relation between $M_{\mathrm{CMO}}$ and galaxy properties, both in slope and normalization.

The fact that our "NSC preservation" model predicts scaling correlations that are consistent with relations involving $M_{\mathrm{CMO}}$ is not coincidental, but rather quite revealing. Indeed, the mass ejected by a $\mathrm{MBH}$ binary is of the order of the mass of the binary itself, with only a weak dependence on the binary's mass ratio and the initial density distribution of stars (Merritt \& Szell 2006). Hence, the mass ejected in $N \mathrm{MBH}$ mergers is approximately $M_{\mathrm{ej}} \approx N M_{\mathrm{MBH}}$, with $M_{\mathrm{MBH}}$ the final MBH mass.

Because infall of gas to the nuclear region can rapidly rebuild a NSC, the relevant quantity to estimate is the number of $\mathrm{MBH}$ mergers since the era at which most of the gas was depleted from the galaxy. Haehnelt \& Kauffmann (2002) compute this quantity using semi-analytic models for galaxy mergers similar to ours. They find that the number of comparable-mass MBH mergers is weakly dependent on galaxy luminosity, and has a small dispersion around a median of 1 , with values $\lesssim 4$ even for the most luminous galaxies. Accordingly, derived mass deficits in "core galaxies" are also found to be peaked around a value of $\approx M_{\mathrm{MBH}}$ Graham 
2004). The total amount of ejected stellar mass is therefore determined mainly by, and is roughly equal to, $M_{\mathrm{MBH}}$. Therefore, $M_{\mathrm{CMO}}=M_{\mathrm{NSC}}+M_{\mathrm{MBH}}$ can be regarded as an approximate estimate (with uncertainties of order a few) of the NSC mass that was in place before mass erosion due to $\mathrm{MBH}$ binaries became important. Our models do indeed confirm this.

These facts explain why the "NSC preservation" model predicts scaling correlations that are consistent with relations involving $M_{\mathrm{CMO}}$, and provide further support for the idea that the broadening/bending of the observed correlations between NSC and host-galaxy properties is a consequence of the nuclear mass erosion caused by $\mathrm{MBH}$ binaries. Finally, the lower panels of Figure 1 show the relations between $M_{\mathrm{CMO}}$ and galaxy properties in the NSC erosion model, for nucleated galaxies with a $\mathrm{MBH}$. Clearly, the choice of plotting the total $M_{\mathrm{CMO}}$ mass significantly reduces the broadening that characterized the $M_{\mathrm{NSC}}$ relations (upper panels), but the scatter is slightly larger than in the "NSC preservation" model (middle panels). This is expected since $M_{\mathrm{ej}}$ only equals the final $\mathrm{MBH}$ mass to first approximation (see equation [1]).

The ancestry of a NSC includes complicated processes leading to its growth, disruption, and regeneration if new material is accreted after a disruptive merger. In Figure2 we illustrate the typical evolution of NSCs as predicted by our fiducial "NSC erosion" model. Since the effect of $\mathrm{MBH}$ binaries and mergers on NSC's is most important for large galaxies (where they cause the broadening/bending of the correlations between NSC and hostgalaxy properties), we choose a galaxy with spheroid mass $\sim 2 \times 10^{10} M_{\odot}$, at which the NSC erosion becomes significant. In this example, the $\mathrm{MBH}$ grows by shortlived accretion events triggered by bar instabilities of the host's galactic disk (at $z \sim 6-7$ ) or by major galactic mergers (at $z \sim 1-3$; note the corresponding growth of the reservoir following the merger-driven infall of cold gas to the nuclear regions). The NSC grows more gradually through a combination of in-situ star formation (especially at $z \sim 1-3$, when the growth of the reservoir triggers nuclear star formation), and infall of stellar clusters. The latter channel dominates over the former at high redshift, and is also enhanced at $z \sim 1-3$, since major galaxy mergers also cause violent bursts of star formation, which in turn enhance stellar-cluster formation. Finally, at $z \lesssim 1-2$ the NSC gets eroded by a series of minor $\mathrm{MBH}$ mergers, but re-forms thanks to a steady infall of stellar clusters, which form as a result of quiescent star-formation activity as the galactic disk re-grows.

Figure 3 shows the redshift evolution of the $M-\sigma$ relations for both $\mathrm{MBHs}$ and $\mathrm{NSCs}$, in the "NSC erosion" model (left panels), and in the "NSC preservation" model (right panels). In both models, the $\mathrm{MBH}$ growth appears inefficient at high redshifts relative to NSC growth. At redshift $z \lesssim 2$, after the epoch of bright quasars, MBHs become the dominant nuclear component in the most massive galaxies. Therefore, it is only at relatively late cosmic epochs that $\mathrm{MBH}$ mergers become efficient at scouring their host clusters, in the "NSC erosion" model. As can be seen, the "NSC preservation" model clearly over-predicts the mass of NSCs in the brightest nucleated spheroids, while the "NSC erosion" model at $z=0$ is in good agreement with the local $M-\sigma$ relation for both MBHs and NSCs.

\section{CONCLUSIONS}

A comparison of our results to observational data reveals that the NSC scaling correlations in the local Universe carry an imprint of the merger and growth history of their companion MBHs. NSCs are significantly eroded by $\mathrm{MBH}$ binaries, causing a broadening of the observed NSC empirical correlations in high-mass galaxies and at low redshifts.

The NSC scaling correlations can potentially be used to probe different NSC evolutionary models, and also to place constraints on the merger and growth history of their host galaxies. However, the slope of the observed correlations involving NSC masses is lowered by the inclusion, at the high- $\sigma$ end, of NSCs that were partly eroded by MBHs. Hence, such relations cannot be used to put reliable constraints on different NSC formation models without also taking into account the scouring effect of $\mathrm{MBH}$ binaries.

We argue that the stellar mass removed from the center during mergers is of order of $M_{\mathrm{MBH}}$, and indeed we find that by replacing $M_{\mathrm{NSC}}$ with $M_{\mathrm{CMO}}=M_{\mathrm{NSC}}+M_{\mathrm{MBH}}$, the observed scaling correlations agree remarkably well with those predicted by models that do not account for the scouring effect of $\mathrm{MBH}$ binaries. Our findings provide a natural explanation to why relations between $M_{\mathrm{CMO}}$ and galaxy properties for nucleated galaxies show significantly less scatter than relations involving $M_{\mathrm{NSC}}$ alone. The relations between $M_{\mathrm{CMO}}$ and galaxy properties are much shallower $\left(\sim \sigma^{2}\right)$ than the same correlations for MBHs $\left(\sim \sigma^{5}\right)$. Our results also explain the well known transition from $\mathrm{MBH}$ - to NSC- dominated galaxies as one proceeds from dwarfs to giant ellipticals, without the need of invoking competitive feedback processes from young NSC's and/or AGN activity. Therefore, the formation of MBHs and NSCs appear not to be mutually exclusive, with NSCs being modified after their formation by the merger evolution of their companion MBHs.

We acknowledge support from a CIERA fellowship at Northwestern University (to F.A.); from the European Union's Seventh Framework Programme (FP7/PEOPLE-2011-CIG) through the Marie Curie Career Integration Grant GALFORMBHS PCIG11-GA2012-321608 (to E.B.); from ERC project 267117 (DARK) hosted by Université Pierre et Marie Curie Paris 6 and at JHU by National Science Foundation grant OIA-1124403 (to J.S.). Computations were performed on SciNet, and on the Horizon Cluster at the IAP.

\section{REFERENCES}

Agarwal, M., \& Milosavljević, M. 2011, ApJ, 729, 35

Aharon, D., \& Perets, H. B. 2015, ApJ, 799, 185

Antonini, F. 2013, ApJ, 763, 62

Antonini, F., Barausse E., \& Silk J. 2015, in preparation
Arca-Sedda, M., \& Capuzzo-Dolcetta, R. 2014, MNRAS, 444 3738

Barausse, E. 2012, MNRAS, 423, 2533; Erratum 2014, MNRAS, 440, 1295 
Bell, E. F., McIntosh, D. H., Katz, N., \& Weinberg, M. D. 2003, ApJS, 149, 289

Bik, A., Lamers, H. J. G. L. M., Bastian, N., Panagia, N., \& Romaniello, M. 2003, A\&A, 397, 473

Böker, T., Laine, S., van der Marel, R. P., et al. 2002, AJ, 123, 1389

Campanelli, M., Lousto, C. O., Zlochower, Y., \& Merritt, D. 2007, Physical Review Letters, 98, 231102

Carson, D. J., Barth, A. J., Seth, A. C., et al. 2015 arXiv:1501.05586

Carollo, C. M., Stiavelli, M., \& Mack, J. 1998, AJ, 116, 68

Côté, P., Piatek, S., Ferrarese, L., et al. 2006, ApJS, 165, 57

den Brok, M., Peletier, R. F., Seth, A., et al. 2014, MNRAS, 445, 2385

Erwin, P., \& Gadotti, D. A. 2012, Advances in Astronomy, 2012

Ferrarese, L., \& Ford, H. 2005, Space Sci. Rev., 116, 523

Ferrarese, L., Côté, P., Dalla Bontà, E., et al. 2006a, ApJ, 644, L21

Georgiev, I. Y., Böker, T. 2014, MNRAS, 441, 3570

Gnedin, O. Y., Ostriker, J. P., \& Tremaine, S. 2014, ApJ, 785, 71

Graham, A. W. 2004, ApJ, 613, L33

Graham A. W. 2012a, MNRAS, 422, 1586

Graham, A. W., \& Spitler, L. R. 2009, MNRAS, 397, 2148

Granato, G. L., De Zotti, G., Silva, L., Bressan, A., \& Danese, L. 2004, ApJ, 600, 580

Gualandris, A., \& Merritt, D. 2008, ApJ, 678, 780

Haehnelt, M. G., \& Kauffmann, G. 2002, MNRAS, 336, L61

Haiman, Z., Ciotti, L., \& Ostriker, J. P. 2004, ApJ, 606, 763

Kormendy, J., \& Ho, L. C. 2013, ARA\&A, 51, 511
Koushiappas, S. M., Bullock, J. S., \& Dekel, A. 2004, MNRAS, 354,292

Kruijssen, J. M. D. 2012, MNRAS, 426, 3008

Krumholz, M. R., McKee, C. F., \& Tumlinson, J. 2009, ApJ, 699, 850; Krumholz, M. R. 2012, ApJ, 759, 9

Madau, P., Haardt, F., \& Dotti, M. 2014, ApJ, 784, LL38

Madau, P., \& Rees, M. J. 2001, ApJ, 551, L27

Merritt, D. 2006, ApJ, 648, 976

Merritt, D., \& Szell, A. 2006, ApJ, 648, 890

Merritt, D. 2013, Dynamics and Evolution of Galactic Nuclei, by David Merritt. Princeton University Press, 2013,

Neumayer, N., \& Walcher, C. J. 2012, Advances in Astronomy, 2012

Parkinson, H., Cole, S., \& Helly, J. 2008, MNRAS, 383, 557

Paturel, G., Petit, C., Prugniel, P., et al. 2003, A\&A, 412, 45

Scott, N., \& Graham, A. W. 2013, ApJ, 763, 76

Sesana, A., Barausse, E., Dotti, M., \& Rossi, E. M. 2014, ApJ 794,104

Seth, A. C., Dalcanton, J. J., Hodge, P. W., \& Debattista, V. P. 2006, AJ, 132, 2539

Tremaine, S. D., Ostriker, J. P., \& Spitzer, L., Jr. 1975, ApJ, 196, 407

Tremaine, S., Gebhardt, K., Bender, R., et al. 2002, ApJ, 574, 740

Turner, M. L., Côté, P., Ferrarese, L., et al. 2012, ApJS, 203, 5

Umemura, M. 2001, ApJ, 560, L29

van Meter, J. R., Miller, M. C., Baker, J. G., Boggs, W. D., \& Kelly, B. J. 2010, ApJ, 719, 1427

Volonteri, M., Lodato, G., \& Natarajan, P. 2008, MNRAS, 383, 1079

Walcher, C. J., van der Marel, R. P., McLaughlin, D., et al. 2005, ApJ, 618, 237 